EDITORA

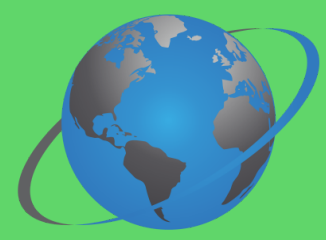

OMNIS SCIENTIA

\title{
SAÚDE PÚBLICA EM
}

\section{TEMPOS PANDEMICOS}

\section{VOLUME 1}

Organizador:

Raul Sousa Andreza

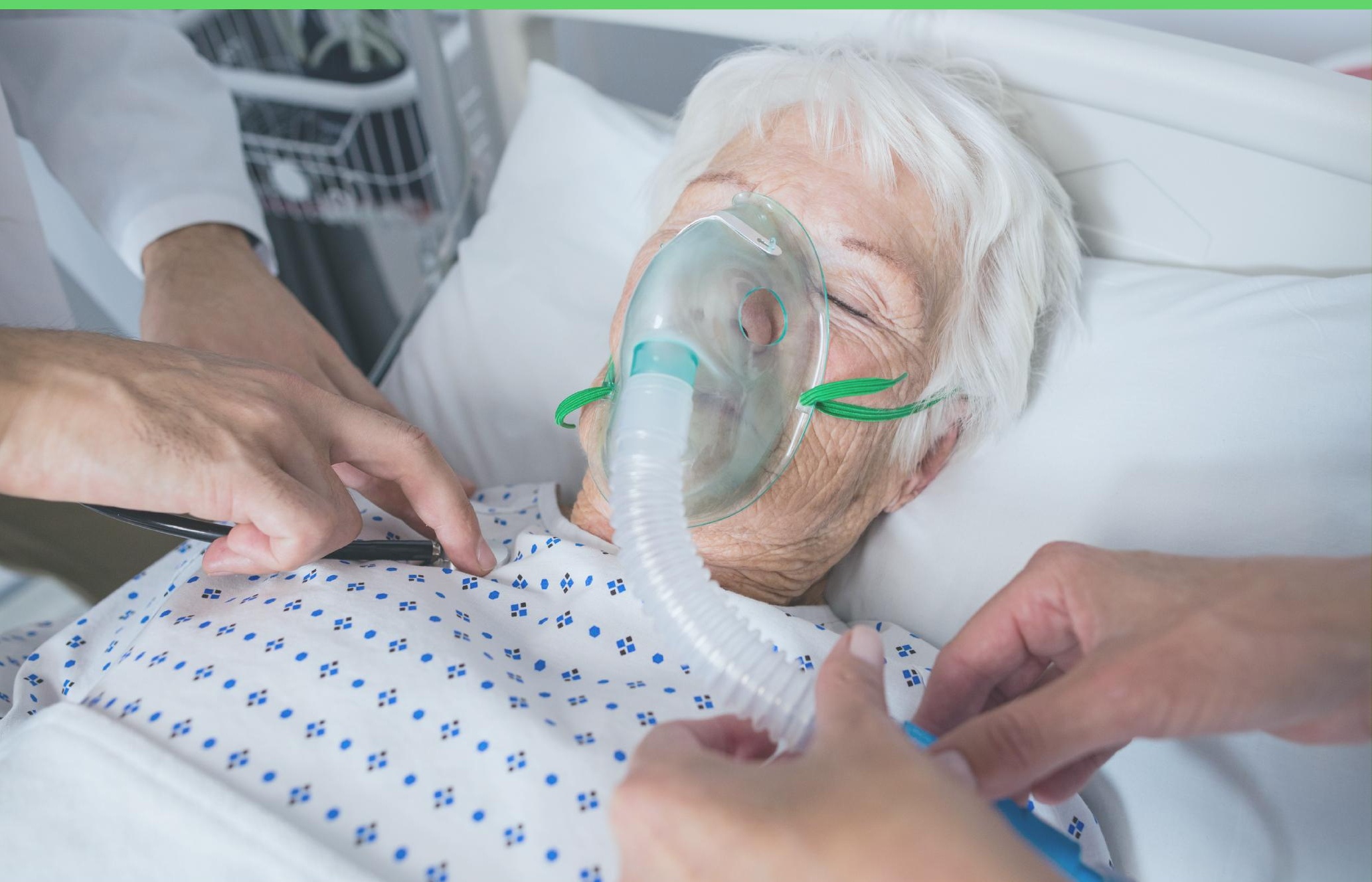


EDITORA

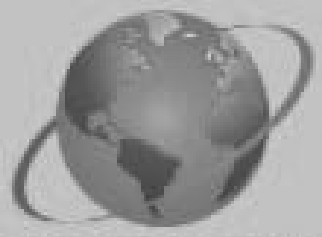

OMNIS SCIENTIA

\section{SAÚDEE PÚBUICA BM}

\section{THEMIPOS PANDENMICOS}

\section{VOLUME 1}

Organizador:

Raul Sousa Andreza

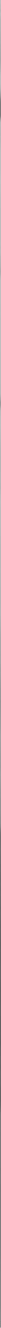


Editora Omnis Scientia

SAÚDE PÚBLICA EM TEMPOS PANDÊMICOS

Volume 1

$1^{a}$ Edição

TRIUNFO - PE

2021 


\section{Editor-Chefe}

Me. Daniel Luís Viana Cruz

Organizador (a)

Prof. Me. Raul Sousa Andreza

\section{Conselho Editorial}

Dra. Pauliana Valéria Machado Galvão

Dr. Wendel José Teles Pontes

Dr. Walter Santos Evangelista Júnior

Dr. Cássio Brancaleone

Dr. Plínio Pereira Gomes Júnior

Editores de Área - Ciências da Saúde

Dra. Camyla Rocha de Carvalho Guedine

Dra. Cristieli Sérgio de Menezes Oliveira

Dr. Leandro dos Santos

Dr. Hugo Barbosa do Nascimento

Dr. Marcio Luiz Lima Taga

Dra. Pauliana Valéria Machado Galvão

\section{Assistentes Editoriais}

Thialla Larangeira Amorim

Andrea Telino Gomes

Imagem de Capa

Freepik

\section{Edição de Arte}

Vileide Vitória Laranjeira Amorim

\section{Revisão}

Os autores

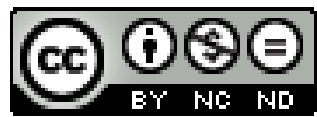

Este trabalho está licenciado com uma Licença Creative Commons - AtribuiçãoNãoComercial-SemDerivações 4.0 Internacional.

O conteúdo abordado nos artigos, seus dados em sua forma, correção e confiabilidade são de responsabilidade exclusiva dos autores. 
Dados Internacionais de Catalogação na Publicação (CIP) (eDOC BRASIL, Belo Horizonte/MG)

S255 Saúde pública em tempos pandêmicos [livro eletrônico] / Organizador Raul Sousa Andreza. - Triunfo, PE: Omnis Scientia, 2021.

286 p. : il.

Formato: PDF

Requisitos de sistema: Adobe Acrobat Reader

Modo de acesso: World Wide Web

Inclui bibliografia

ISBN 978-65-88958-41-4

DOI 10.47094/978-65-88958-41-4

1. Ciências da saúde. 2. Saúde pública. 3. Pandemia. I. Andreza, Raul Sousa.

CDD 610

Elaborado por Maurício Amormino Júnior - CRB6/2422

\author{
Editora Omnis Scientia \\ Triunfo - Pernambuco - Brasil \\ Telefone: +55 (87) 99656-3565 \\ editoraomnisscientia.com.br \\ contato@editoraomnisscientia.com.br
}




\section{PREFÁCIO}

A incidência do novo coronavírus no Brasil é preocupante. No entanto, a saúde pública do país e o sistema de atendimento visa abranger a diversidade que o Brasil apresenta, o sistema único de saúde (SUS) tem como base a integralidade, a universalidade e a equidade de todos os pacientes e trabalhadores. Instituído assim, para democratizar toda a saúde brasileira, tem o interesse de ofertar serviços de qualidade a população. Portanto, ao longo da história de sua consolidação a saúde pública foi deixado de lado e passou a gerar grandes problemas aos profissionais atuantes.

De fato, os estudos desenvolvidos no âmbito da saúde pública se propõem a articular conhecimentos de diferentes saberes e fazeres fornecendo subsídios teóricos, práticos e metodológicos que contribuem positivamente para a construção de estratégias e políticas públicas que visam o desenvolvimento de informações e ações em prol de uma saúde de qualidade para toda comunidade.

O presente livro é composto por 24 capítulos elaborados por autores pertencentes às ciências da saúde e suas áreas afins com o objetivo de proporcionar conhecimentos e compartilhar experiências e resultados de estudos desenvolvidos em várias localidades brasileiras e que visam à elucidação de diferentes situações de saúde.

Em nossos livros selecionamos um dos capítulos para premiação como forma de incentivo para os autores, e entre os excelentes trabalhos selecionados para compor este livro, o premiado foi o capítulo 02, intitulado "VACINAS CONTRA COVID-19: UMA BREVE DESCRIÇÃO POR MEIO DE REVISÃO INTEGRATIVA“. 


\section{SUMÁRIO}

CAPÍTULO 1

A IMPORTÂNCIA E OS DESAFIOS DA ESTRATÉGIA DE SAÚdE DA FAMÍLIA NO ENFRENTAMENTO DA COVID-19

Romana Erica Tavares Grangeiro Pinto

Wyara Ferreira Melo

Maria Amanda Laurentino Freires

Patrício Borges Maracajá

Aline Carla de Medeiros

José Cândido da Silva Nóbrega

Manoel Marques de Souto Nóbrega Filho

Túlio Alberto de Oliveira Sousa

Mônica Valéria Barros Pereira

Vicente Saraiva dos Santos Neto

Hozanna Estrela Celeste

Gabriela Rocha Pordeus dos Santos

DOI: 10.47094/978-65-88958-41-4/17-26

CAPÍTULO 2

VACINAS CONTRA COVID-19: UMA BREVE DESCRIÇÃO POR MEIO DE REVISÃO INTEGRATIVA

Fernanda Lima Marçal

Isabela Figueiredo e Souza

Maria Eduarda Coelho Gomes

Larissa Lima Torres

Isabela Campbell Santos

Thamara Lóren Lima

Ludmilla Vieira Magalhães

Maria Eduarda Sirina Pereira

Lucas Viana de Oliveira 
Larissa da Silva Torres França

Natan Fiorotti da Silva

Milena de Oliveira Simões

DOI: 10.47094/978-65-88958-41-4/27-41

CAPÍTULO 3

AÇÃO EM SAÚdE E A IMPORTÂNCIA DO USO CORRETO DAS MÁSCARAS PARA PREVENÇÃO DE INFECÇÕES RESPIRATÓRIAS

Soniely Nunes de Melo

Rafael Belarmino de Souza Lima

Tarcísio Correia Sposito

Rayana Ribeiro Trajano de Assis

Nayara Sandriele Santana de Souza

Bruna Rafaella Santos Torres

Flávio José Alencar de Melo

Davi Silva de Jesus

Carlos Henrique Bezerra de Siqueira

Izabelle Barbosa da Silva

Marcos André de Holanda Prudente Pessoa

Ana Marlusia Alves Bomfim

DOI: 10.47094/978-65-88958-41-4/42-51

CAPÍTULO 4

CONHECIMENTOS DAS EQUIPES MULTIPROFISSIONAIS DA ESTRATÉGIA DE SAÚDE DA FAMIILIA SOBRE COVID-19 E GESTAÇÃO

Priscilla dos Santos Nascimento

Michelle Araújo Moreira

DOI: 10.47094/978-65-88958-41-4/52-65 
CARTILHA EDUCATIVA SOBRE A COVID-19 PARA A PROTEÇÃO DA COMUNIDADE ACADÊMICA DO CAMPUS BELÉM DO IFPA

Lidineusa Machado Araujo

Maria de Nazaré Pereira Rodrigue Martins

Gabriela Priscila de Lima Carvalho

Fernanda Rafaela de Souza Rebelo da Costa

Michelle da Silva Pereira

Andréa de Melo Valente

Maria Helena Cunha Oliveira

Antônio Marcos Mota Miranda

DOI: 10.47094/978-65-88958-41-4/66-75

CAPÍTULO 6 .76

A SAÚdE DOS PROFISSIONAIS DE SAÚDE QUE ATUAM NA LINHA DE FRENTE NA PANDEMIA DO COVID - 19: REVISÃO INTEGRATIVA

João Lucas Ferreira Andrade

Léa Bianch Lima

Luana Kellen Nogueira Epitácio

Maria Eduarda Alves Vasconcelos

Antônio Augusto Ferreira Carioca

Carlos Antônio Bruno da Silva

Eudóxia Sousa de Alencar

DOI: 10.47094/978-65-88958-41-4/76-89

CAPÍTULO 7 .90

OS EFEITOS DA PANDEMIA NA COMPULSÃO ALIMENTAR: UMA REVISÃO DE LITERATURA

Lais Pontes de Miranda Cerqueira

Tarcio Goncalves sobral

Isadora Bianco Cardoso de Menezes

DOI: 10.47094/978-65-88958-41-4/90-97 
O IMPACTO DA PANDEMIA DO COVID - 19 SOBRE A SEGURANÇA ALIMENTAR E NUTRICIONAL: REVISÃO INTEGRATIVA

Bruna Sousa Barbosa

Igor Matheus Cruz de Oliveira

João Lucas Ferreira Andrade

Léa Bianch Lima

Luana Kellen Nogueira Epitácio

Maria Eduarda Alves Vasconcelos

Antônio Augusto Ferreira Carioca

Carlos Antônio Bruno da Silva

Eudóxia Sousa de Alencar

DOI: 10.47094/978-65-88958-41-4/98-104

CAPÍTULO 9 105

A UTILIZAÇÃO DOS PROTOCOLOS DE USO DO GUIA ALIMENTAR PARA A POPULAÇÃO BRASILEIRA: RELATO DE EXPERIÊNCIA

Bárbara Santos Amorim

Lis Chaves Marinho

Isadora Bianco Cardoso de Menezes

DOI: 10.47094/978-65-88958-41-4/105-114

CAPÍTULO 10 .

O IMPACTO DA COVID-19 EM PORTADORES DE SÍNDROME METABÓLICA

Laura Rasul de Lima

Ana Beatriz Amaral Vieira

Gabriella Neiva Reis

Ingrid Ravenna Liberalino Lima

DOI: 10.47094/978-65-88958-41-4/115-122 
SIMPLIFICANDO A HANSENÍASE: PROJETO DE INTERVENÇÃO REALIZADO EM

MEIO DIGITAL DURANTE A PANDEMIA

Amanda Almeida Lima

Ana Beatriz Sousa Santos

Francisco Vittor Miranda e Araújo

Jesamar Correia Matos Filho

João Ferreira de Paula Neto

Maria Clara de Freita Albano

Manoel Cícero Viana de Lima

Pedro Schmitt Martins Paiva Matos

Ruddy Mariano Maia Cysne Guerra

Samuel Carvalho Vasconcelos

Thaine Mirla Rocha

Elaine Lopes Bomfim

DOI: 10.47094/978-65-88958-41-4/123-131

CAPÍTULO 12.

DESCOMPLICANDO A HANSENÍASE: PROJETO DE INTERVENÇÃO REALIZADO EM UNIDADE DE SAÚDE NO PERÍODO DA PANDEMIA

Adália Stefanny de Araujo Cavalcante

Giovanna Giffoni Souza do Nascimento

Iêda de Freitas Martins Jota

Isabel Camila Araújo Barroso

Kaio Rangel Freitas Guimarães

Laís Mesquita de Sousa

Monique dos Santos Chaves

Manoel Victor Freires Vieira

Matheus Macedo Braga Coelho

Thaine Mirla Rocha

Elaine Lopes Bomfim 
CAPÍTULO 13. 140

HANSENÍASE: UM ESTUDO EPIDEMIOLÓGICO NA REGIÃO DO MÉDIO ARAGUAIAMATO GROSSO

Flavia Rodrigues Santana

Josilene Dália Alves

DOI: 10.47094/978-65-88958-41-4/140-150

CAPÍTULO 14 151

MUNICÍPIOS COM ALTA INCIDÊNCIA DE TUBERCULOSE EM MATO GROSSO: CONHECER PARA INTERVIR EM TEMPOS DE PANDEMIA

Larissa Machado Bellé

Yasmim Paloma Abreu Silva

Alessandro Rolim Scholze

Josilene Dália Alves

DOI: 10.47094/978-65-88958-41-4/151-162

CAPÍTULO 15 163

SAÚDE INTEGRAL DA MULHER EM CONTEXTO PANDÊMICO: REVISÃO INTEGRATIVA DE LITERATURA

Lohana Guimarães Souza

Tailande Venceslau Carneiro

Letícia Grazielle Santos

DOI: 10.47094/978-65-88958-41-4/163-175

CAPÍTULO 16 176

ASSISTÊNCIA DE ENFERMAGEM NO OUTUBRO ROSA E PERFIL EPIDEMIOLÓGICO DAS MULHERES: RELATO DE EXPERIÊNCIA

Helena Pereira de Souza

Laura Letícia Perdição Guerra

Luana Fernandes e Silva 
Thales Philipe Rodrigues da Silva

Alessandra Lage Faria

Helen Carine Ferreira Balena

Érica Moreira de Souza

Bruna Luíza Soares Pinheiro

Lorena Medeiros de Almeida Mateus

Flávia Duarte de Oliveira Ribeito

Bianca Maria Oliveira Luvisaro

Fernanda Penido Matozinhos

DOI: 10.47094/978-65-88958-41-4/176-188

CAPÍTULO 17 189

AGRAVAMENTO DAS DOENÇAS PSIQUIÁTRICAS DURANTE O PERÍODO DE ISOLAMENTO SOCIAL: UMA BREVE REVISÃO DE LITERATURA

Hellen Kristina Magalhães Brito

Gabriela Teixeira Lima

Ana Laura Fernandes Tosta

Laura Beatriz Caitano de Oliveira

Maria Paula Ricardo Silva

Mariana Vieira Garcia de Carvalho

Nathália Siriano Costa

Mayara Rita Figueredo

Mabel Fernandes Rocha

Helena Maria Mendes Marques

Kaio Murilo Santana Corrêa

Ana Flávia Buiatte Andrade

DOI: 10.47094/978-65-88958-41-4/189-200 
ATUAÇÃO DO PSICÓLOGO EM TEMPO DE PANDEMIA: UMA REVISÃO DE ESTUDOS NACIONAIS

Gabriel Rigamonte

Sueli Souza

Wilson Quiroz

Daniel Bartholomeu

Fernando Pessotto

Cintia Heloína Bueno

Fernanda Helena Viana Garcia

DOI: 10.47094/978-65-88958-41-4/201-213

CAPÍTULO 19

CRIAÇÃO DE UM APLICATIVO VOLTADO PARA UMA UNIDADE BÁSICA DE SAÚdE EM FORTALEZA

Isabella Araujo Duarte

Giovanna Rolim Pinheiro Lima

Idna Lara Goes de Sena

Laura Figueiredo Leite

Letícia Cavalcante Lócio

Livian Araújo Camelo Gomes

Maria Regina Cardoso Linhares Oliveira Lima

Maria Tereza Linhares Cardoso

Pedro Henrique Cardoso Nogueira

Rafael Albuquerque Franco

Rodrigo Carvalho Paiva

Berta Augusta Faraday Sousa Pinheiro

DOI: 10.47094/978-65-88958-41-4/214-229 
TELEMEDICINA E SUAS VARIÁVEIS NO CENÁRIO DE PANDEMIA MUNDIAL: UMA REVISÃO DE LITERATURA

Raniere Canteiro Garcia Lhamas

Andressa Marcolino Campos

Douglas Ferreira Lima

Gabriel Souza Ferreira Oliveira

Guilherme de Mendonça Lopes Beltrão

Luciana de Paula Santana

Nícollas Nunes Rabelo

DOI: 10.47094/978-65-88958-41-4/230-237

CAPÍTULO 21 238

MONITORIA ACADÊMICA NO ENSINO REMOTO: PERSPECTIVAS E DESAFIOS EM TEMPOS PANDÊMICOS

Felipe Gabriel Assunção Cruz

Givanildo Bezerra de Oliveira

Marcílio Delan Baliza Fernandes

Ana Lúcia Moreno Amor

DOI: 10.47094/978-65-88958-41-4/238-248

CAPÍTULO 22

A DOENÇA DO OLHO SECO NA SÍINDROME DE SJÖGREN

Bruna Rafaella Santos Torres

Carlos Eduardo Ximenes da Cunha

Carlos Henrique Bezerra de Siqueira

Flavia Emanuelly Alves França Gomes

Santília Tavares Ribeiro de Castro e Silva

Anna Caroline Guimarães Gomes

Laís Rytholz Castro

Dennis Cavalcanti Ribeiro Filho 
Lara Medeiros Pirauá de Brito

Marina Viegas Rezende Ribeiro

DOI: 10.47094/978-65-88958-41-4/249-259

CAPÍTULO 23 260

FEIRAAGROECOLÓGICA: DIFICULDADES E POTENCIALIDADES DE UM CIRCUITO CURTO DE COMERCIALIZAÇÃO

Maria Rita Garcia de Medeiros

Rônisson Thomas de Oliveira Silva

Maria Natalícia de Lima

Ana Beatriz Macêdo Venâncio dos Santos

DOI: 10.47094/978-65-88958-41-4/260-269

CAPÍTULO 24 270

AVALIAÇÃO DO IMPACTO DE UMA REDE SOCIAL EM USUÁRIOS DE UMA UNIDADE BÁSICA DE SAÚDE EM FORTALEZA

Luiz Gerson Gonçalves Neto

Letícia Cavalcante Lócio

Carlos Alexandre Leite Pereira Filho

Henrique Sousa Costa

Maria Helena dos Santos Macedo

Lígia Bringel Olinda Alencar

Berta Augusta Faraday Sousa Pinheiro

Isaac Dantas Sales Pimentel

DOI: 10.47094/978-65-88958-41-4/270-280 


\section{CAPÍTULO 18}

ATUAÇÃO DO PSICÓLOGO EM TEMPO DE PANDEMIA: UMA REVISÃO DE

ESTUDOS NACIONAIS

\section{Gabriel Rigamonte';}

Setor de Psicologia Centro Universitário Padre Anchieta (UNIANCHIETA), Jundiaí, São Paulo. http://lattes.cnpq.br/9324474797891897

\section{Sueli Souza ${ }^{2}$;}

Setor de Psicologia Centro Universitário Padre Anchieta (UNIANCHIETA), Jundiaí, São Paulo. http://lattes.cnpq.br/7653119957401824

\section{Wilson Quiroz}

Setor de Psicologia Centro Universitário Padre Anchieta (UNIANCHIETA), Jundiaí, São Paulo. http://lattes.cnpq.br/3320877001618921

\section{Daniel Bartholomeu';}

Nexo - Instituto de Psicologia Aplicada / Setor de Psicologia Centro Universitário Padre Anchieta (UNIANCHIETA), Jundiaí, São Paulo.

\section{http://lattes.cnpq.br/1327488708059314}

\section{Fernando Pessotto5;}

Nexo - Instituto de Psicologia Aplicada / Setor de Psicologia Centro Universitário Padre Anchieta (UNIANCHIETA), Jundiaí, São Paulo.

http://lattes.cnpq.br/9932025382224441

\section{Cintia Heloína Bueno ${ }^{6}$;}

Nexo - Instituto de Psicologia Aplicada.

http://lattes.cnpq.br/1403005793921426

\section{Fernanda Helena Viana Garcia?}

Nexo - Instituto de Psicologia Aplicada.

RESUMO: O presente trabalho promove uma revisão bibliográfica da literatura nacional com o objetivo de agrupar e sintetizar os conhecimentos produzidos a fim de facilitar o acesso aos psicólogos que estão atuando em campo no enfretamento a Covid-19, fornecendo ferramentas que auxiliam a compreensão de estratégias de planejamento do atendimento on-line. Para tanto foi feita uma vasta pesquisa levando em consideração os parcos estudos já efetivados com relação à COVID-19 sendo contextualizados com estudos de pandemias anteriores, foi descrito também os problemas de saúde 
mental desencadeados na população em geral e nos profissionais de saúde que trabalham na linha de frente contra a COVID-19. Tais estudos e definições serviram de ponto inicial para uma compreensão e assimilação da presente realidade social, emocional, econômica e profissional que toda a população e os profissionais de psicologia estão expostos. Ao final do artigo foi descrito o que é e como funciona o atendimento remoto, modalidade que não é nova, porém adquiriu protagonismos ante este cenário pandêmico.

PALAVRAS-CHAVE: Saúde Mental. Intervenção psicológica. Teleatendimento. Pandemia.

\section{PSYCHOLOGIST'S PERFORMANCE IN TIME OF PANDEMIC: A REVIEW OF NATIONAL STUDIES}

ABSTRACT: The present chapter aim at a literature review of the national production of psychologists actuation in COVID-19 in Brazil in order to facilitate access to psychologists who are working in the field to confront Covid-19, providing tools that help to understand online service planning strategies. For So much research has been done taking into account the meager studies already carried out with respect to COVID-19 being contextualized with previous pandemic studies, it was also described the mental health problems triggered in the general population and in the health professionals working on the front lines against COVID-19. Such studies and these definitions served as a starting point for an understanding and assimilation of this social, emotional, economic and professional reality that the entire population and professionals of psychology are exposed. At the end of the article it was described what it is and how it works remote attendance, a modality that is not new, but has gained prominence pandemic scenario.

KEY-WORDS: Mental health. Psychological intervention. Call center. Pandemic.

\section{INTRODUÇÃO}

A doença COVID-19 foi identificada em dezembro de 2019, apresentando um rápido crescimento, com isso instituições governamentais de saúde mobilizaram planos de prevenção, urgência e emergência para conter o aumento de casos e a sobrecarga nos serviços de atendimento a população. Dentre os planos de medidas de distanciamento social, como, por exemplo, suspensão das atividades escolares, confinamento doméstico, incentiva à realização do trabalho remoto e restrições de viagens, fechamento de indústrias e comércios, entre outros. Essas medidas causam bastante impacto na economia, relações sociais, saúde mental das pessoas, convivência familiar, pois essas medidas não só influencia no avanço da doença, o que também merece total atenção pelos profissionais da saúde (Aiello-Vaisberg, T. M. J, Gallo-Belluzzo, S. R., \& Visintin, C.; 2020).

Segundo (Brooks et al., 2020) no contexto da epidemia do Covid-19, alguns dos principais estressores estão relacionados à duração da quarentena, distanciamento social, frustração e tédio, acúmulo de tarefas, como, por exemplo, realização de atividades que normalmente são feitas fora de 
casa, agora são incluídas dentro de sua casa (homeschooling e homeworking, por exemplo), também o medo de contrair a doença, ao estigma da doença, trabalho de risco no caso de trabalhadores da saúde e serviços vitais, preocupação com a própria saúde e entes queridos (Enumo, S. R. F., Niederauer, J, \& de Lara, W.; 2020).

Nos recentes meses pandêmicos houve um grande número de estudos e pesquisas que abordaram as questões da atuação do psicólogo ante os diversos problemas na saúde mental desencadeados pelo isolamento social. Este estudo abordou a problemática "Como estão às pesquisas da atuação do psicólogo durante a pandemia no Brasil?". Neste sentido a presente pesquisa teve como objetivo agrupar e sintetizar os conhecimentos produzidos a fim de facilitar o acesso aos psicólogos que estão atuando em campo no enfretamento a Covid-19, fornecendo ferramentas que auxiliam a compreensão de estratégias de planejamento do atendimento on-line, tanto na preparação e resposta para as populações em geral, pacientes infectados, familiares e trabalhadores da linda de frente. Este artigo de revisão bibliográfica tem caráter importante no Brasil, uma vez que revela elementos falhos e estudos brasileiros com relação às sequelas da pandemia e sua relevância está na sintetize de estudos e fornecimento parâmetros para comparação com estudos estrangeiros.

\section{MÉTODOLOGIA}

Esta foi uma pesquisa de revisão bibliográfica de natureza aplicada que objetivou "gerar conhecimentos para aplicação prática dirigida à solução de problemas específicos" (Prodavoe \& Freitas, 2013, p.51).

Os procedimentos adotados foram: primeiramente foi delimitado o tema e a questão norteadora; na sequência foi feita uma pesquisa de publicações pertinentes ao tema; leitura e fichamento das informações; e por fim a elaboração e construção do presente artigo. A figura a seguir é um fluxograma com processo metodológico utilizado na elaboração da pesquisa para o presente artigo:

Figura 1: Fluxograma da metodologia de pesquisa.

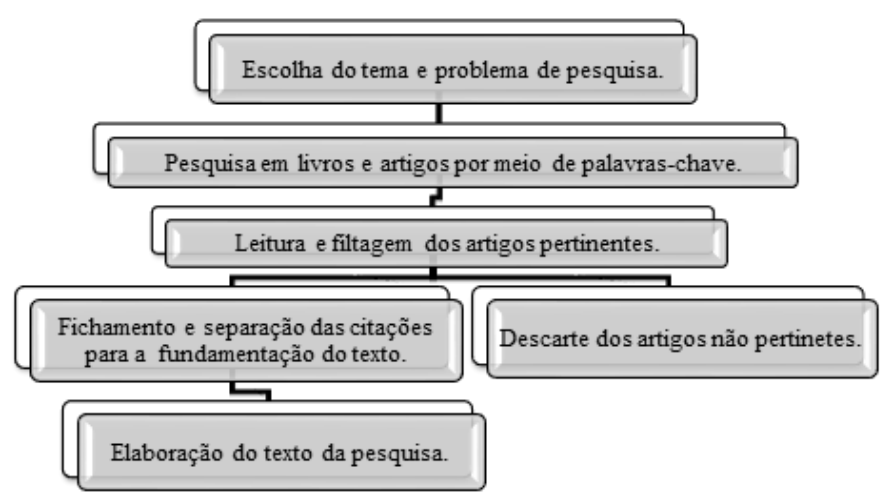

Fonte: Autoria própria. 
A pesquisa dos artigos foi realizada entre os meses de julho a outubro de 2020, os quais foram filtrados em dois idiomas (português e inglês) com datas de publicações entre os anos de 2008 e, majoritariamente, 2020. A escolha desta linha temporal adveio da necessidade de fazer uma corelação entre os problemas da saúde mental ocasionados pela pandemia de Covid-19 e como ocorreu em surtos edêmicos anteriores, tais como o Sars e Ebola, a fim de fornecer elementos completares aos parcos estudo já realizado sobre a pandemia de Covid-19.

Para a busca dos estudos científicos foram utilizados como palavras-chave os termos: Covid-19; Coronavirus, Saúde Mental; Papel do psicólogo na pandemia; teleatendimento durante a pandemia. Depois da busca, foram considerados relevantes os artigos de universidades renomadas, sites acadêmicos, portais Governamentais e de Serviço de Saúde que se fornecem informações pertinentes à pergunta norteadora. Foram descartados estudos de publicações não catalogadas, que fossem de anos inferiores a 2008 e que não apresentaram pertinência para o presente estudo.

\section{RESULTADOS E DISCUSSÕES}

Neste estudo foram encontrado um total de 35 trabalhos pertinentes ao objetivo agrupar e sintetizar os conhecimentos produzidos a fim de facilitar o acesso aos psicólogos que estão atuando em campo no enfretamento a Covid-19, destes, 11 estão hospedados no Scielo, 12 foram retirados do PubMed e 13 de sites institucionais do governo (CAPES). Para melhor compreensão, foram sintetizadas na tabela a seguir as publicações encontradas por meio das palavras chaves e que se mostraram pertinentes a este estudo de acordo com cada base de dados:

Tabela 1: Número de trabalhos encontrados por base de dados

\begin{tabular}{|c|c|c|c|}
\hline Base de dados & Pandemia de Covid-19 & Saúde mental & Teleatendimento \\
\hline Scielo & 06 & 02 & 03 \\
\hline PubMed & 05 & 04 & 03 \\
\hline CAPES & 02 & 08 & 03 \\
\hline Total: & 12 & 14 & 09 \\
\hline
\end{tabular}

Fonte: autoria própria.

Os resultados da pesquisa bibliográfica de estudos científicos que abordavam a questões de saúde mental, atendimento psicológico e a pandemia de Covid-19 trouxeram indagações significativas sobre a atuação profissional do psicólogo no presente cenário. Os artigos pesquisados trouxeram a dados sobre os impactos socioeconômicos e sociais na população, elementos estes muito citados como desencadeadores e agravantes de fobias, estresses, ansiedade.

Outro elemento observado por meio da pesquisa nos artigos foi que, diante das restrições de isolamento impostas pelo Estado par o combate à propagação da doença, houve uma diminuição do acesso às políticas públicas de saúde e de proteção dos casos de violência doméstica. Os principais 
afetados foram àquelas famílias de baixa renda que tiveram um grande comprometimento na renda e como tem uma grande defasagem de acesso às tecnologias de informações (TICs) se viram com suporte de assistência social ainda mais reduzido.

Diante de contexto de isolamento social, o atendimento remoto adquiriu ainda mais protagonismo, desta forma as publicações oficiais das agencias governamentais e do Conselho Federal de Psicologia foram no sentido de regulamentara e orientar os atendimentos e diagnósticos.

Para a sistematização desta pesquisa e facilitação do entendimento das discussões, após a leitura e análise crítica os estudos selecionados como pertinentes a esta pesquisa foi feita uma organização segundo os objetivos aos quais eles se relacionam. Esta organização de dados está representada no quadro a seguir:

Quadro 1: Distribuição das referencias por eixo temático da pesquisa.

\begin{tabular}{|c|c|}
\hline Eixos temáticos da pesquisa: & eferências: \\
\hline $\begin{array}{l}\text { A pandemia decorrente da Covid-19 } \\
\text { e as questões relacionadas à Saúde } \\
\text { Mental }\end{array}$ & $\begin{array}{l}\text { Aiello-Vaisberg, T. M. J, Gallo-Belluzzo, S. R., \& Visintin, C.; (2020); Brooks } \\
\text { et al., (2020); Hall, R. C. W., Hall, R., \& Chapman, M. J. (2008); Jiang et al., } \\
\text { (2020); Schmidt, B., Crepaldi, M. A., BOLZE, S. D. A., Neiva-Silva, L., \& } \\
\text { Demenech, L. M. (2020); Spink (2020); Taylor (2019); Zanon, C., Zanon, L. } \\
\text { L. D., Weschler, S. M., Fabretti, R. R., \& da Rocha, K. N.; (2020) }\end{array}$ \\
\hline $\begin{array}{l}\text { As consequências na Saúde Mental } \\
\text { decorrentes do isolamento social }\end{array}$ & $\begin{array}{l}\text { Bao, Sun, Meng, Shi, \& Lu, (2020); Crepaldi, M. A., Schmidt, B., da Silva } \\
\text { Noal, D., Bolze, S. D. A., \& Gabarra, L. M; (2020); FIOCRUZ (2020); Kang } \\
\text { et al., (2020); Li et al., (2020); Reger; Stanley; Joiner, (2020); UNICEF } \\
\text { (2020); Zandifar \& Badrfam, (2020). }\end{array}$ \\
\hline $\begin{array}{l}\text { Os cuidados com a Saúde Mental } \\
\text { durante a pandemia de Covid-19 e o } \\
\text { papel do psicólogo neste processo. }\end{array}$ & $\begin{array}{l}\text { BRASIL (2020); Dores, A., Barbosa, F., \& Silva, R. (2017); Conselho Federal } \\
\text { de Psicologia. (2020); Crestana, T. (2015). FIOCRUZ (2020); Fleury, H. J. } \\
\text {; (2020); Goyal et al., (2020); IBGE (2020); IPEA (2020); Pereira, B. P., } \\
\text { Soares, C. R. S., Galvani, D., da Silva, M. J., de Almeida, M. C., Bianchi, } \\
\text { P. C., \& Barreiro, R. G.; (2020); Pieta, M. A. M., \& Gomes, W. B.; (2014) } \\
\text { Siqueira, C. C. A., Simon, R., \& Russo, M. N.; (2014); Tokarina (2020); } \\
\text { Weinberg, (2020). }\end{array}$ \\
\hline
\end{tabular}

Fonte: autoria própria.

Estes eixos temáticos apresentados no quadro acima delimitam a fundamentação teórica utilizada nas sessões das discussões a seguir.

\section{A pandemia decorrente da Covid-19 e as questões relacionadas à Saúde Mental}

Ainda é cedo para vislumbrar panorama completo de todas as implicações que a pandemia do COVID-19 desencadeará, todavia pesquisas anteriores sobre outros surtos infecciosos revelaram agravamentos nos problemas socioemocionais e mentais, a curto, médio e longo prazo, em toda a população geral e para os profissionais da saúde (Jiang et al., 2020;). Sendo assim é de se esperar iminentes diagnósticos de problemas emocionais e mentais em um grande numero de pessoas, e, em 
especial, em profissionais que trabalharam na chamada "linha de frente", que são aqueles profissionais que trabalham diretamente com as pessoas contaminadas com a doença (Li et al., 2020a).

Pesquisas realizadas após a epidemia de ebola, em 1995, demonstram que a população em geral temia pela morte e pelo afastamento enquanto os profissionais de saúde descreveram o grande medo de se contaminar com a doença, e um medo ainda maior de transmiti-la a familiares, o sofrimento pelo afastamento da família somado a fadiga, sensação de perda de controle e a preocupação por não saber quanto tempo duraria a epidemia foram agravantes para o aumento do estresse sofrido (Hall et al., 2008). Após epidemia de 2003 de SARS (Síndrome Respiratória Aguda Grave), que é outro tipo de coronavirus, as decorrências psicológicas superaram o número de ocorrências médicas com relação ao numero geral de afetados e a duração dos sintomas (Taylor, 2019). Outros fatores de sofrimento mental foram com relação à duração do período de quarentena e relacionados às condições de trabalho tais como a frustração, falta de suprimentos e informações inadequadas sobre a doença.

Diante de tantas implicações e agravantes no campo da psique, os profissionais que tratam da saúde mental adquirem um protagonismo, talvez não desejado, mas necessário. Desta forma foi necessário todo um processo de reestruturação de processos de atendimento a fim se adequar a nova realidade de afastamento social, para tanto foi publicado a Resolução CFP n ${ }^{\circ}$ 4/2020 em 26 de março de 2020, a qual permite a prestação de serviços psicológicos por meio de tecnologias da informação e comunicação para aquelas pessoas em situações situação de urgência, emergência e desastre, bem como de violação de direitos ou violência. As situações de urgência e emergência podem ser definidas de acordo com:

\footnotetext{
A Resolução do Conselho Federal de Medicina n ${ }^{\circ}$ 1.451, de 17 de março de 1995, estabelece nos parágrafos I e II do artigo $1^{\circ}$, as definições para os conceitos de urgência e emergência a serem adotas na linguagem médica no Brasil. Define, ainda no parágrafo primeiro, que urgência é a "ocorrência imprevista de agravo à saúde com ou sem risco potencial de vida, cujo portador necessita de assistência médica imediata". No parágrafo segundo, a definição de emergência aparece como "a constatação médica de condições de agravo à saúde que impliquem em risco iminente de vida ou sofrimento intenso, exigindo, portanto, tratamento médico imediato". (Brasil, 2015, p. 282)
}

É importante também levar em consideração a violação de direito humanos uma vez que pesquisas têm mostrado que crianças e mulheres estão desprotegidas durante essa situação de confinamento, trazendo consigo várias limitações, entre as quais se inclui a dificuldade de pedir ajuda. Nesse sentido, os índices de aumento de violência contra crianças e mulheres, podendo resultar em um grande aumento do número de feminicídios que ocorrem durante esse período de convivência forçada (Aiello-Vaisberg, T. M. J. Gallo-Belluzzo, S. R., \& Visintin, C.; 2020).

Sendo assim foi fundamental tais resoluções para permitir a flexibilização da forma como são feitos os atendimentos com intuito de minimizar as implicações psicológicas diante da COVID-19 (CFP, 2020a). A Resolução CFP n ${ }^{\circ}$ 4/2020 suspende, durante o período de pandemia do COVID-19, os Art. $3^{\circ}, 4^{\circ}, 6^{\circ}, 7^{\circ}$ e $8^{\circ}$ da Resolução CFP n ${ }^{\circ} 11 / 2018$ (Schmidt et al, 2020). Estas implicações e 
dificuldades serão detalhadas mais a frente neste artigo.

\section{As consequências na Saúde Mental decorrentes do isolamento social}

Um dos dilemas com relação aos problemas na saúde mental está no fato deste problema ser muito discriminado e "o sofrimento psicológico, com frequência, é visto como uma fraqueza, algo que a pessoa teria condições de resolver, mas não o faz" (Fiocruz, 2020, p3). Todavia há muito tempo que ocorre um gradativo crescimento de doenças e patologias ligadas a saúde mental na população em geral, "segundo a Organização Mundial da Saúde, estima-se que os transtornos depressivos unipolares estão em terceiro lugar na classificação da carga global de adoecimentos" (Brasil, 2015, P.27). Tais doenças são muito perigosas uma vez que na maior parte das vezes passam despercebidas ou são tratadas com leviandade por aqueles que convivem com o individuo.

A crise em saúde mental pode ser considerada um episódio de desestabilização específica em que o sujeito parece não dar conta das intensidades afetivas que lhe perpassam naquele momento, impedindo tanto a própria pessoa, quanto aqueles de seu convívio, de levarem sua vida cotidiana. (BRASIL, 2015, p.285).

O advento da pandemia de COVID-19 e o isolamento social agravam os problemas relacionados à saúde mental "pois a vida não é o que se passa apenas em cada um dos sujeitos, mas principalmente o que se passa entre os sujeitos, nos vínculos que constroem e que os constroem como potência de afetar e ser afetado" (BRASIL, 2010, p. 8), desta forma o que ocorre na sociedade afeta em demasia o indivíduo em suas particularidades. As incertezas sobre a Covid-19 e a mudança na rotina podem causar estresse, ansiedade, depressão, raiva, sobrecarga emocional, sono de má qualidade e até piora na saúde física.

\section{A saúde mental dos profissionais que trabalham na linha de frente}

A orientação geral é que as pessoas procurem ficar em casa e evitem aglomerações. Todavia os profissionais de saúde fazem parte de um grupo de serviços essenciais e são fundamentais num cenário pandêmico, desta forma enquanto as demais pessoas se resguardam, estes profissionais trabalham pelo bem geral. Dentro do ambiente clínico todos os materiais podem ser repostos, novos respiradores comprados e os leitos reorganizados. Mas os profissionais não são objetos, por isso os "trabalhadores e trabalhadoras da saúde são os recursos mais preciosos a serem preservados e a receber suporte durante e pós-pandemia" (Fiocruz, 2020, p.2).

São muitos os elementos estressantes que os profissionais têm que lidar tal como o risco de contrair a doença, difíceis decisões com relação à triagem e tratamento dos pacientes, somado ao luto e dor da perda sucessiva tanto de pacientes quanto de colegas e, em alguns casos, de familiares. A pressão, o estresse, as incertezas e as cobranças em demasia durante eventos de pandemia podem comprometer a atenção, concentração e as decisões dos profissionais, problemas que afetam o bem 
estar dos profissionais e que podem continuar tendo efeito mesmo após o fim do surto de COVID-19 (Kang et al., 2020).

Um problema que deve ser observado com atenção e cuidado é o suicídio, uma vez que é fenômeno complexo e multifatorial (Fiocruz, 2020), e o advento da pandemia multiplica os fatores de sofrimento na população em geral. Estudos mostram que entre os profissionais de saúde existe uma taxa de expressiva de suicídio, desta forma é necessário também que haja estratégias preventivas e de suporte voltadas aos profissionais que atuam na linha de frente para estarem preparados para tantos desafios e pressões (Reger; Stanley; Joiner, 2020).

\section{Os cuidados com a Saúde Mental Durante a Pandemia de Covid-19 e o Papel do Psicólogo neste} Processo

Os conhecimentos científicos da área da Psicologia tem grande relevância no sentido do entendimento da pandemia, suas consequências para a saúde mental e social dos indivíduos. Desta forma, tais conhecimentos podem servir como base para a construção de medidas e ações preventivas voltadas para a população com relação aos efeitos psicológicos ocasionados pela pandemia. Estas medidas trazem também subsídios e recomendações para o trabalho dos profissionais da saúde. Tais medidas foram sistematizadas no quadro a seguir:

Quadro 2: medidas preventivas de acordo com os conhecimentos científicos da psicologia.

\begin{tabular}{|c|l|l|}
\hline \multicolumn{3}{|c|}{ Medidas preventivas de acordo com os conhecimentos científicos da psicologia } \\
\hline I & Acesso a informações, claras, coesas e uteis. & $\begin{array}{l}\text { O acesso a informações confiáveis e a compreensão das mesmas diminuo o } \\
\text { estresses, a possibilidade de ansiedade e depressão decorrentes de do medo } \\
\text { e desinformação. }\end{array}$ \\
\hline II & Comunicação com órgãos de saúde pública & $\begin{array}{l}\text { O conhecimento das medidas de proteção e prevenção contra a Covid-19 } \\
\text { promovem um auto cuidado nos indivíduos e um trabalho coletivo na } \\
\text { prevenção da doença }\end{array}$ \\
\hline III & $\begin{array}{l}\text { Cuidados e apoio para os pacientes em } \\
\text { isolamento social }\end{array}$ & $\begin{array}{l}\text { Proporcionando meios para o atendimento e diagnostico rápido em caso de } \\
\text { infecção. Propiciando informações, apoio, orientações e cuidados. }\end{array}$ \\
\hline IV & Atendimento e suporte psicossocial & $\begin{array}{l}\text { Ampliação de atendimentos com tratamento preventivo e estímulo a hábitos } \\
\text { saudáveis dentro de casa, de forma a prevenir possíveis problemas de Saúde } \\
\text { Mental. }\end{array}$ \\
\hline
\end{tabular}

Fonte: adaptado de Pereira et al, 2010.

\section{As tecnologias de informação e comunicação e o teletratamento}

O Conselho Federal de Psicologia (CFP) que regulamenta a Telepsicologia no Brasil, pela RESOLUCÃO Nº 011/2012 reconhece cinco modalidades de telepsicologia, a saber: as Orientações Psicológicas de diferentes tipos, entendendo-se por orientação o atendimento realizado em até 20 encontros ou contatos virtuais, síncronos ou assíncronos; os processos prévios de Seleção de Pessoal; a Aplicação de Testes devidamente regulamentados por resolução CFP No 005/2012; a Supervisão do trabalho de psicólogos, realizada de forma eventual ou complementar ao processo de sua 
formação profissional presencial e o Atendimento Eventual de clientes em trânsito e/ou de clientes que momentaneamente se encontrem impossibilitados de comparecer ao atendimento presencial. No Brasil, o psicólogo que realiza a Telepsicologia regularmente, é obrigado a realizar o cadastramento desses serviços e do site no Conselho Regional de Psicologia (CRP). (Siqueira, C. C. A., Simon, R., \& Russo, M. N.; 2014).

De um modo geral, psicólogos e pacientes se adaptam bem até mesmo a uma apresentação visual relativamente distorcida, porém problemas relacionados com o som não são muito bem tolerados, com isso criando desafios metodológicos para o terapeuta, como, por exemplo, o psicólogo tem que desenvolver novas habilidades de comunicação e uma nova proposta de formação do profissional, para superar as múltiplas ocorrências técnicas possíveis (Judge et al., 2011). Ao aderir a telepsicologia, o psicoterapeuta irá encontrar questões de limite, improváveis no cenário tradicional do encontro presencial, pois limites apropriados garantem os objetivos terapêuticos, por isso é provável que surjam bastantes desafios nessa nova modalidade, pela pouca familiaridade, porém (Weinberg, 2020) discute alguns obstáculos para essa transição da sessão psicodramática para uma prática temporariamente denominada telepsicodrama (Fleury, H. J. ; 2020).

Pacientes relataram que, ao se adaptar ao vídeo, a relação com o terapeuta diferiu das relações face a face, mas não necessariamente de modo pior, ou melhor, onde a sensibilidade experiencial de estudos qualitativos anotou oscilações comunicais que requerem atenção na relação terapêutica online, pois a experiência por videoconferência apresentou uma maior dificuldade dos terapeutas interpretarem a linguagem corporal dos pacientes, para estabelecer relação empírica, terem um contato olho no olho (Simpson, Bell, Knox, \& Mitchell, 2005). Em outro estudo, de acordo com (Himle et al., 2006), os pacientes disseram que se sentiam na sala do terapeuta, se acomodaram rapidamente ao vídeo, a maior parte dos estudos investiga a efetividade de terapias cognitivo-comportamental (TCC) online, elas têm se mostrado efetivas no tratamento de depressão, transtorno do pânico, ansiedade, estresse pós-traumático e fobia (Pieta, M. A. M., \& Gomes, W. B.; 2014).

$\mathrm{Na}$ terapia online, quando se questiona, é preciso entender a capacidade de se desenvolver uma relação terapêutica à distância, alguns relatos e estudos de atendimento têm se mostrado ser possível estabelecer um vínculo através desse método, com resultados, por vezes, semelhantes entre os tratamentos presenciais e a distância (Pereira, B. P., Soares, C. R. S., Galvani, D., da Silva, M. J., de Almeida, M. C., Bianchi, P. C., \& Barreiro, R. G.; 2020).

É preciso um melhor aprofundamento nos estudos para compreender e avaliar a relação terapêutica nas formas de psicoterapia pela internet, no entanto, a literatura vem crescendo nesse sentido, por exemplo, os estudos de (Hanley), entrevistou adolescentes que receberam atendimento psicoterápico via e-mail em um site da terapia do Reino Unido, e de (Joraas, Rimehaug, Birkeland e Arefjord, 2009) que entrevistaram três pacientes adolescentes que terminaram o atendimento presencial 


\section{CONSIDERAÇÕES FINAIS}

A presente pesquisa trouxe uma sistematização de conhecimentos sobre as consequências do isolamento social imposto pela pandemia de Covid-19 e seus efeitos na Saúde Mental da população em geral e nos profissionais da Saúde. A pandemia provocada pelo Covid-19 é considerada a maior emergência de saúde que o mundo enfrentou nas ultimas décadas, neste cenário compreende-se que os profissionais que cuidam da Saúde Mental têm um papel fundamental no enfrentamento da doença, na conscientização da população e no cuidado das doenças desencadeadas pelo isolamento social.

Os atendimento e intervenções realizados de forma remota durante o momento pandêmico foi uma ferramenta que ajudou a minimizar os impactos negativos na vida dos indivíduos, proporcionando uma melhora na saúde mental de todos. Posteriormente ao termino da pandemia estes atendimentos tem caráter essencial na readaptação à vida social, assimilação das mudanças e de como lidar com as perdas.

Observou-se uma lacuna não abordada pelos estudos analisados no sentido de um acesso democrático dos atendimentos, uma vez que a aqui já foi citado às dificuldades da população mais carente de acessarem as TICs, este público que está sujeito tanto quanto, ou talvez até mais, que a população mais abastada a serem acometidos de doenças de cunho mental. Neste sentido, esta é uma área de pesquisa a ser abordada e estudada pelos cientistas e acadêmicos a fim de proporcionar recursos para uma melhora nos atendimentos a esta parcela da população.

Outro campo ainda a ser mais estudado é as consequências na saúde mental dos trabalhadores da linha de frente do combate à pandemia. Estudos de surtos anteriores permitem um vislumbre das consequências do estresse e sobrecarga acumulados por razão do prolongado momento pandêmico, estima-se que as reais proporções só poderão ser estudadas com maior aprofundamento nos meses subesquentes a retomada total da normalidade.

As pesquisas realizadas até o presente momento trazem conhecimentos relevantes para a atuação do profissional psicólogo, todavia, tendo em conta que a pandemia ainda não chegou ao fim, tais pesquisas apresentam limitações sobre a real amplitude dos problemas de saúde mental que serão desencadeados pelo isolamento social. Estima-se que nos meses após a reabertura total do convívio social e da retomada das atividades muitas pessoas que se mantiveram isoladas por medo ou por serem do grupo de risco e que não tem acesso ou conhecimentos sobre as tecnologias da informação buscarão atendimento para sanar problemas decorrentes do prolongado isolamento.

Conclui-se este estudo salientando a importância do cuidado com a saúde mental dos indivíduos durante e após o isolamento social. Neste cenário, o profissional psicólogo como elemento chave na prevenção e cuidados dos sofrimentos mentais e possíveis transtornos psiquiátricos decorrentes da pandemia.

\section{DECLARAÇÃO DE INTERESSES}

Nós, autores deste artigo, declaramos que não possuímos conflitos de interesses de ordem financeira, comercial, político, acadêmico e pessoal. 


\section{REFERÊNCIAS}

Aiello-Vaisberg, T. M. J., Gallo-Belluzzo, S. R., \& Visintin, C. Maternidade e Sofrimento Social em Tempos de Covid 19: Estudo de Mommy Blogs.

BRASIL. Ministério da Saúde. Secretaria de Atenção à Saúde. Núcleo Técnico da Política Nacional de Humanização. Acolhimento nas práticas de produção de saúde. 2. ed. 5. reimp. Brasília, 2010.

.Ministério da Saúde. Portaria n. 356. Dispõe sobre a regulamentação e operacionalização do disposto na Lei $n^{\circ} 13.979$, de 6 de fevereiro de 2020, que estabelece as medidas para enfrentamento da emergência de saúde pública de Importância. Diário Oficial da União, 49. http://www.in.gov.br/ web/dou/-/portaria-n-356-de-11-de-marco-de-2020-247538346

. Ministério da Saúde. Secretaria de Atenção à Saúde. Departamento de Ações Programáticas Estratégicas. Saúde Mental / Ministério da Saúde, Secretaria de Atenção à Saúde, Departamento de Ações Programáticas Estratégicas. - Brasília: Ministério da Saúde, 2015. 548 p.: il. (Caderno HumanizaSUS ; v. 5)

. MINISTERIO DA SAUDE. Saúde Sem Fake News. MS, 2020. Disponível em: <https:// www.saude.gov.br/fakenews>.

Bao, Y., Sun, Y., Meng, S., Shi, J., \& Lu, L. (2020). 2019-nCoV epidemic: address mental health care to empower society. The Lancet, 395(10224), e37-e38. http://dx.doi.org/10.1016/S01406736(20)30309-3

Brooks, B. L., Iverson, G. L., Sherman, E. M., \& Roberge, M. C. (2010). Identifying cognitive problems in children and adolescents with depression using computerized neuropsychological testing. Applied Neuropsychology, 17, 37-43.

Conselho Federal de Psicologia. (2020). Resolução do exercício profissional nº4, de 26 de março de 2020. Dispõe sobre regulamentação de serviços psicológicos prestados por meio de Tecnologia da Informação e da Comunicação durante a pandemia do COVID19. Disponível em: https://atosoficiais. com.br/cfp/resolucao-do-exercicio-profissional-n-4-2020-dispoe-sobre-regulamentacao-de-servicospsicologicos-prestados-por-meio-de-tecnologia-da-informacao-e-da-comunicacao-durante-apandemia-do-covid19?origin=instituicao.

Crepaldi, M. A., Schmidt, B., da Silva Noal, D., Bolze, S. D. A., \& Gabarra, L. M. Terminalidade, Morte e Luto na Pandemia de COVID-19: Demandas Psicológicas Emergentes e Implicações Práticas.

Crestana, T. (2015). Novas abordagens terapêuticas-terapias on-line. Revista Brasileira de Psicoterapia, 17(2), 35-43.

Dores, A., Barbosa, F., \& Silva, R. (2017). Theraphy 2.0: chegar mais perto dos que estão longe= Therapy 2.0: getting closer to those who are far.

Enumo, S. R. F., Niederauer, J., \& de Lara, W. Enfrentando o estresse em tempos de pandemia: Proposição de uma cartilha1, 2, 3. 
Fleury, H. J. (2020). Psicodrama e as especificidades da psicoterapia on-line. Revista Brasileira de Psicodrama, 28(1), 1-4.

FIOCRUZ/FGV. Cartilha Processo de Luto no Contexto da COVID-19. Série: Saúde e Mental e Atenção Psicossocial na Pandemia Covid-19.

. Cartilha Recomendações aos Psicólogos para o Atendimento Online. Série: Saúde e Mental e Atenção Psicossocial na Pandemia Covid-19.

.Cartilha Suicídio na Pandemia. Série: Saúde e Mental e Atenção Psicossocial na Pandemia Covid-19.

.Cartilha Trabalhadores da Saúde. Série: Saúde e Mental e Atenção Psicossocial na Pandemia Covid-19.

.Cartilha Violência Doméstica e Familiar na COVID-19. Série: Saúde e Mental e Atenção Psicossocial na Pandemia Covid-19.

Goyal, K., Chauhan, P., Chhikara, K., Gupta, P., \& Singh, M. P. (2020). Fear of COVID 2019: first suicidal case in India. Asian Journal of Psychiatry, 49(101989). http://dx.doi.org/10.1016/j. ajp.2020.101989

Hall, R. C. W., Hall, R., \& Chapman, M. J. (2008). The 1995 Kikwit Ebola outbreak: lessons hospitals and physicians can apply to future viral epidemics. General Hospital Psychiatry, 30(5), 446-452. http://dx.doi.org/10.1016/j. genhosppsych.2008.05.003

IBGE. Uso da Internet, televisão e celular no Brasil. Disponível em: https://educa. ibge.gov.br/jovens/materias-especiais/20787-uso-de-internet-televisao-e-celular-no-brasil.html. Acesso em 29 de setembro de 2020.

IPEA, Instituto de Pesquisa Econômica Aplicada. Nota Técnica/Prevenindo conflitos sociais violentos em tempos de pandemia: garantia de renda, manutenção da saúde mental e comunicação efetiva.

Jiang, X., Deng, L., Zhu, Y., Ji, H., Tao, L., Liu, L., Ji, W. (2020). Psychological crisis intervention during the outbreak period of new coronavirus pneumonia from experience in Shanghai. Psychiatry Research, 286, 112903. http://dx.doi. org/10.1016/j.psychres.2020.112903

Kang, L. et al. The mental health of medical workers in Wuhan, China dealing with the 2019 novel coronavirus. The Lancet Psychiatry, v. 7, n. 3, p. e14, 1 mar. 2020.

Li, Z., Ge, J., Yang, M., Feng, J., Qiao, M., Jiang, R., Yang, C. (2020a). Vicarious traumatization in the general public, members, and non-members of medical teams aiding in COVID-19 control. Brain, Behavior, and Immunity. http:// Dx.Doi.Org/10.1016/J.Bbi.2020.03.007

Pereira, MD, Oliveira, LC, Costa, CFT, Bezerra, CMO, Pereira, MD, Santos, CKA \& Dantas, EHM (2020). The COVID-19 pandemic, social isolation, consequences on mental health and coping strategies: an integrative review. Research, Society and Development, 9(7): 1-35, e652974548. 
Pereira, B. P., Soares, C. R. S., Galvani, D., da Silva, M. J., de Almeida, M. C., Bianchi, P. C., \& Barreiro, R. G. Terapia Ocupacional Social: reflexões e possibilidades de atuação durante a pandemia da Covid-19/Social Occupational Therapy-reflections and possibilities for action the COVID-19 pandemic. Revista Interinstitucional Brasileira de Terapia Ocupacional-REVISBRATO, 4(3), 554566.

Pieta, M. A. M., \& Gomes, W. B. (2014). Psicoterapia pela Internet: viável ou inviável?. Psicologia: Ciência e profissão, 34(1), 18-31.

Reger, M. et al. Suicide Mortality and Coronavirus Disease 2019 - A Perfect Storm? JAMA Psychiatry, 2020. Disponível em: https://jamanetwork.com/journals/jamapsychiatry/fullarticle/2764584. Acesso em: 07 de setembro de 2020.

Schmidt, B., Crepaldi, M. A., BOLZE, S. D. A., Neiva-Silva, L., \& Demenech, L. M. (2020). Impactos na Saúde Mental e Intervenções Psicológicas Diante da Pandemia do Novo Coronavírus (COVID-19).

Singulane, B. A. R., \& SARTES, L. M. A. (2017). Therapeutic Alliance in Cognitive Behavior Therapy for Videoconferencing: A Literature Review. Psicologia: Ciencia e Profissao, 37(3), 784.

Siqueira, C. C. A., Simon, R., \& Russo, M. N. TELEPSICOLOGIA NO BRASIL-Desafios e Novas Perspectivas.

Spink, Mary J. P. Fique em casa: a gestão de riscos em contexto de incerteza. PSICOLOGIA \& SOCIEDADE, 32, e020002. Programa de Estudos Pós-graduados em Psicologia Social, Pontifícia Universidade Católica de São Paulo, SP, 2020. Disponível em: http://dx.doi.org/10.1590/18070310/2020v32239826.

Taylor, S. The psychology of pandemics: preparing for the next global outbreak of infectious disease. Newcastle upon Tyne: Cambridge Scholars Publishing, 2019.

Prodanov, Cleber Cristiano; Freitas Ernani Cesar. Metodologia do trabalho científico: métodos e técnicas da pesquisa e do trabalho acadêmico. - 2. ed. - Novo Hamburgo: Feevale, 2013.

Tokarina, Mariana. (2020) Um em cada 4 brasileiros não tem acesso à internet, mostra pesquisa Número representa 46 milhões que não acessam a rede. Agência Brasil - Rio de Janeiro, 2020. Disponível em: https://agenciabrasil.ebc.com.br/economia/noticia/2020-04/um-em-cada-quatrobrasileiros-nao-tem-acesso-internet. Acesso em 29 de setembro de 2020.

Weinberg, R. A. (2020). Intelligence and IQ: Landmark issues and great debates. American Psychologist, 44, 98-104.

Zandifar, A., \& Badrfam, R. (2020). Iranian mental health during the COVID-19 epidemic. Asian Journal of Psychiatry, 51, 101990. http://dx.doi.org/10.1016/j.ajp.2020.101990

Zanon, C., Zanon, L. L. D., Weschler, S. M., Fabretti, R. R., \& da Rocha, K. N. COVID-19: Implicações e aplicações da Psicologia Positiva em tempos de pandemia. 


\section{Índice Remissivo}

$\mathbf{A}$

Acesso aos psicólogos 201, 203, 204

Acesso às redes sociais 43

Agricultor e o consumidor 260

Agricultores familiares 260, 263, 266

Alterações do metabolismo 115

Ambiente virtual 215

Ansiedade 77, 78, 81, 82, 83, 84, 86, 87, 91, 92, 94, 95, 117, 121, 190, 192, 193, 194, 197, 204, 207, 208, 209

Aplicativo 136, 215, 217, 218, 219, 220, 221, 222, 223, 224, 225, 226, 227, 228, 243, 271, 272, 273, 274, 278, 280

Aprendizagem 239

Assistência social 163, 171, 205

Associação da covid-19 com a gestação 52

Astrazeneca (universidade de oxford) 28, 30

Atenção primária 25, 124

Atenção primária à saúde (aps) 19, 59, 107, 125, 177, 178

Atendimento ambulatorial 105

Atendimento on-line 201, 203

Atendimento remoto 202, 205

Atividades econômicas 28

Autoanticorpos 250

Autoridades sanitárias 67, 69, 73, 278

C

Capacidade de defesa do organismo 115

Características dos imunizantes 28, 30

Cartilha educativa sobre a covid-19 67, 69, 70

Ceratoconjuntivite sicca $(\mathrm{kcs}) 250$

Combate à hanseníase 133

Comercialização 38, 260, 261, 262, 263, 264, 265, 266, 267, 268, 269

Compulsão alimentar 90, 92, 93, 94, 95, 96

Consumo de alimentos 105

Contaminação 43, 44, 45, 47, 49, 72, 73, 74, 78, 117, 135, 179, 191, 193, 195, 197

Coronavac (sinovac) 28, 29

Cuidados individuais e coletivos $67,69,73$

Cuidados preventivos ao covid-19 115

D

Depressão 78, 81, 82, 83, 87, 91, 92, 94, 95, 110, 172, 190, 192, 193, 194, 197, 207, 208, 209

Desemprego 21, 99, 103, 153

Desenvolvimento do câncer 177, 185

Desigualdade em saúde 163

Desinformação 29, 208 
Diabetes 21, 56, 57, 95, 105, 106, 107, 108, 114, 116, 117, 118, 119, 120, 181, 183, 186

Direito humano à alimentação adequada 103, 260

Disseminação da informação 67,73

Distanciamento social 25, 48, 55, 61, 68, 73, 90, 95, 126, 130, 192, 195, 202, 279

Distúrbios psicológicos 190

Doença infecciosa 53, 125, 133, 134, 140, 141, 151, 152

Doença multifatorial 250

Doenças 21, 24, 28, 29, 43, 54, 56, 57, 59, 69, 91, 95, 106, 107, 108, 115, 116, 117, 119, 120, 129, 133, 137, 140, 142, $149,161,163,164,167,171,172,173,177,185,186,193,194,195,207,210,217,222,223,227,250,271$, $272,273,278,279$

Doenças crônicas 105, 171, 218, 221, 227, 273

Doenças negligenciadas 140, 142

\section{$\mathbf{E}$}

Educação em saúde 19, 24, 43, 46, 48, 50, 67, 69, 73, 105, 108, 133, 134, 135, 136, 137, 145, 179, 185, 215, 216, 217, $227,228,238,240,248,271,272,274$

Efeitos colaterais $28,30,31,32,33$

Enfermagem 50, 56, 57, 58, 60, 61, 88, 89, 122, 149, 161, 176, 177, 178, 180, 188, 227, 240, 280

Enfermidade epidêmica 238

Enfrentamento da covid-19 18, 19, 20, 23, 24, 25

Epidemiologia 114, 140, 148, 149, 160, 161, 242, 248

Equipamentos de proteção 24, 43, 47, 78, 171, 197

Equipes multiprofissionais $52,54,55,56,59,60,61,62$

Estratégia saúde da família (esf) 18, 19, 23, 54

Estratégias nutricionais 105

Estresse 77, 78, 81, 82, 83, 86, 87, 88, 89, 94, 172, 190, 192, 193, 197, 206, 207, 209, 210, 211

Etiologia autoimune 250

Evidências científicas 29, 52, 56, 57, 59, 61, 62, 63, 76, 79, 99, 101, 190

F

Feiras agroecológicas 260, 261, 264

Ferramenta tecnológica de saúde 215

Fortalecimento da saúde púbica 67

Frequência cardíaca 230

G

Gestação 52, 53, 54, 57, 58, 59, 61, 62, 63, 224

Glândulas exócrinas 250, 251, 252, 253

Guia alimentar 105, 107, 108, 112, 113

$\mathbf{H}$

Hábitos alimentares 90, 92, 94, 95, 111, 112

Hanseníase 124, 125, 126, 127, 128, 130, 131, 133, 134, 135, 136, 137, 138, 139, 140, 141, 142, 143, 144, 145, 146, $147,148,149$

Herança genética 115

Hipertensão 57, 95, 105, 107, 108, 114, 115, 116, 118, 119, 120, 121, 181, 183, 186, 228, 229, 274, 276,278 
Hiposecreção lacrimal 250

I

Idosos 19, 21, 23, 54, 105, 108, 134, 137

Impacto da pandemia 99

Imunização 28, 29, 30, 35, 38, 179, 183, 186

Inclusão 20, 22, 52, 55, 56, 58, 75, 77, 79, 101, 163, 165, 167, 215, 226, 268

Infecções 28, 29, 38, 43, 44, 48, 57, 59, 60, 78, 165, 172, 180, 227, 251

Infecções respiratórias virais $43,44,48$

Informação de qualidade 271, 279

Informação em saúde 67,69

Informação sobre a hanseníase 124

Instabilidade econômica 99, 103

Instrumento de prevenção 43

Intervenção psicológica 202

Isolamento $23,48,55,60,62,81,90,91,92,94,100,142,164,177,179,190,191,192,193,194,195,196,197,198$, $199,203,204,205,207,208,210,242,261$

Isolamento social 23, 26, 62, 90, 92, 94, 100, 142, 164, 177, 179, 190, 191, 192, 194, 195, 196, 197, 198, 203, 205, 207, 208, 210, 242

$\mathbf{J}$

Janssen (johnson \& johnson) 28, 29, 30

$\mathbf{L}$

Lesões cutâneas 140, 143

Linha de frente 76, 78, 79, 81, 84, 86, 87, 88, 171, 195, 197, 202, 206, 207, 208, 210

M

Má qualidade do sono 77,87

Máscaras faciais 43

Material digital 43, 46

Medição biométrica 230

Medidas de isolamento 26, 28, 194

Medidores de glicose 230

Metodologia pico (problema, intervenção, contexto, resultado) 76, 79, 99, 101

Monitores do centro de ciências da saúde (ccs) 238

Monitoria 238, 239, 240, 241, 242, 243, 244, 246

Morbidade 115, 119

Mortalidade materna 163, 171, 172

Mudanças nos estilos de vida 90, 92, 93

Mycobacterium leprae 135, 140, 141, 146

N

Níveis socioeconômicos 43, 46

Novas modalidades de interações 238

Novo coronavírus sars-cov-2 18, 19 
Olho seco 250, 251, 252, 253, 254, 255, 256, 257, 258

Organização mundial de saúde (oms) 19, 52, 53, 54, 68, 92, 191

Organização pan-americana de saúde (opas) 52, 54

Órgãos oficiais de saúde 238, 247

Outubro rosa $177,178,179,184,185$

$\mathbf{P}$

Padrões dietéticos 115

Pandemia 18, 19, 20, 21, 23, 24, 25, 26, 28, 31, 38, 45, 47, 48, 53, 55, 68, 69, 73, 75, 76, 78, 79, 81, 82, 84, 86, 87, 88, $89,90,91,92,93,94,95,96,99,100,101,102,103,104,117,118,119,120,124,126,130,131,133,135,136$, $137,142,152,153,160,161,162,163,164,165,168,169,170,171,172,173,174,177,179,180,186,187$, 190, 191, 192, 193, 194, 195, 196, 197, 198, 199, 200, 203, 204, 205, 206, 207, 208, 210, 211, 212, 213, 238, $239,240,241,242,244,247,248,271,272,279,280$

Perfil alimentar 105

Perfil clínico 160, 177, 183, 185

Pfizer (pfizer e biontech) 28, 30

Políticas públicas de saúde 18, 24, 173, 204

Portadores de síndrome metabólica 115

Prática oftalmológica 250

Práticas do autocuidado 178,186

Pressão arterial 115,230

Prevenção 29, 33, 72, 170, 187, 221

Primeiros socorros 215, 218, 219, 225, 227, 273

Princípio ativo 28, 30, 33

Produção de alimentos 260

Profissionais de psicologia 202

Profissionais de saúde 18, 22, 24, 54, 56, 76, 78, 79, 81, 82, 84, 85, 86, 87, 89, 107, 125, 138, 151, 160, 170, 185, 194, $195,197,198,202,206,207,208,238$

Programa nacional de alimentação escolar (pnae) 99, 265

Promoção em saúde 43, 45, 271, 278

Propagação do vírus $43,47,48,67,69$

Q

Qualidade de vida 22, 47, 82, 106, 112, 155, 217, 226, 244, 250, 251, 254, 272, 278

Quarentena 90, 100, 121, 126, 170, 172, 190, 191, 192, 193, 194, 195, 199, 202, 206

$\mathbf{R}$

Recurso tecnológico 230

Redes sociais 43, 45, 46, 49, 133, 137, 194, 271, 272, 274, 278, 279

Responsabilidade individual e coletiva 18, 24

Restrição social 90, 92, 93 
Sars-cov- $20,21,29,30,31,32,33,34,35,38,39,40,45,49,53,56,57,58,59,60,65,88,120,163,164,165,168$, $173,191,199,238,239,240,242,243,247,248,279$

Saúde da família 18, 19, 20, 22, 23, 24, 25, 26, 52, 54, 55, 62, 64, 125, 135, 179, 187

Saúde da mulher 163, 166, 173

Saúde de maneira remota 133

Saúde dos profissionais $76,79,81,86,89$

Saúde dos trabalhadores $77,86,87$

Saúde integral 52, 163, 165, 167, 171

Saúde mental 78, 83, 88, 90, 93, 94, 95, 96, 172, 179, 190, 191, 192, 193, 194, 195, 196, 197, 198, 202, 203, 204, 206, $207,208,210,212,222,227,279$

Saúde psicológica 77,87

Saúde pública $6,20,28,38,69,73,82,120,125,134,139,141,148,149,160,168,169,170,171,172,177,179,197$, $199,208,211,238,240,242,247$

Secura ocular 250, 251, 257

Sedentarismo 94, 107, 115, 179

Segurança alimentar e nutricional 99, 100, 101, 102, 103, 104

Segurança alimentar nutricional (san) 99

Serviço de saúde 71, 115, 120, 180

Síndrome de sjögren (ss) 250, 251

Síndrome metabólica 115, 116, 117, 118, 119, 120

Sistema imunológico 58, 115, 117, 120

Sistema nacional de agravos de notificação (sinan) 140, 143

Situação de vulnerabilidade 90, 94, 95, 103

Superfície ocular 250, 254, 258

T

Tecnologia 66, 67, 69, 70, 137, 211, 231, 244

Tecnologias de informação e comunicação 208, 230

Teleatendimento 202, 204

Telemedicina 133, 170, 230, 232

Telessaúde 230, 236

Tempos pandêmicos 271

Terapêutica multidisciplinar 190

Teste de schirmer 250, 255

Testes oftalmológicos 250

Transmissão de informações 271, 279

Transtorno de ansiedade generalizada 190, 192

Tuberculose (tb) 151,152

$\mathbf{U}$

Unidade de saúde da família (usf) 52, 55

Uso das máscaras 43, 45, 48 
Vacina 28, 31, 32, 33, 34, 37, 39, 40, 70, 72, 159, 161, 183, 184, 186, 221

Variantes $28,30,31,34,35,38,49,78$

Vídeos educativos 215, 226

Violência contra a mulher $163,169,170,173,174$

$\mathbf{X}$

Xeroftalmia 250,251 


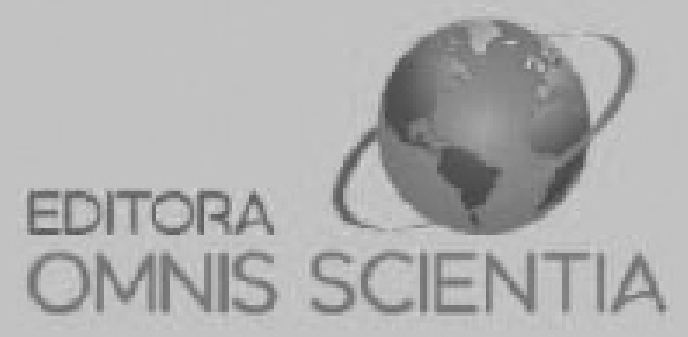

editoraomnisscientia@gmail.com M https://editoraomnisscientia.com.br/ @editora_omnis_scientia (0) https://wwww.facebook.com/omnis.scientia.9 $\boldsymbol{\oplus}$ +55 (87) 9656-3565 (C) 


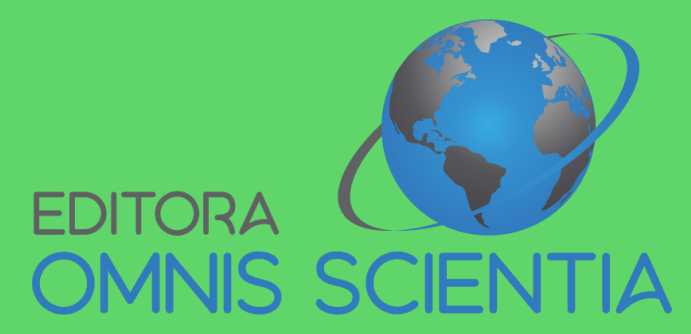

editoraomnisscientia@gmail.com M https://editoraomnisscientia.com.br/ (-) @editora_omnis_scientia (0) https://www.facebook.com/omnis.scientia.9 $\subsetneq$ +55 (87) 9656-3565 @ 